\title{
Out of Hospital Cardiac Arrest: A Case Report
}

\author{
Kenan Ljuhar ${ }^{1 *}$, Adem Zalihić ${ }^{1}$, Aida Gavranović ${ }^{1}$, Basri Lenjani², \\ Premtim Rashiti ${ }^{2}$, Nuhi Arslani ${ }^{3}$
}

Received: 28 July 2020 / Accepted: 22 August 2020 / Published online: 20 July 2021

(C) The Author(s) 2021. This article is published with open access at https://journal.astes.org.al

\begin{abstract}
Introduction: Sudden OHCA(Out of hospital cardiac arrest) is the third leading cause of death in industrialized nations. With more than $60 \%$ of cardiovascular deaths resulting from cardiac arrest, it remains the leading cause of death worldwide. Heart rhythms associated with cardiac arrest are divided into two groups: shockable rhythms (ventricular fibrillation/pulseless ventricular tachycardia (VF/ pVT) and non-shockable rhythms (asystole and pulseless electrical activity (PEA)). VF is the most commonly identified arrhythmia in cardiac arrest patients. Urgent medical treatment includes cardiopulmonary resuscitation and early defibrillation.

Material and Methods: Materials for this case report are data collected from the medical records of the Emergency Medical Center of Sarajevo protocol of patients.

Case report: Our case report is presented with 59 years old man who had OHCA in his apartment. The initial rhythm was VF, and cardiopulmonary resuscitation was provided due to the Advanced life support guidelines to shockable rhythms. It was delivered 3 DC Shock-s (200J, 300J, $360 \mathrm{~J}$ ) with the biphasic defibrillator, it was administered 1mg Adrenalin and performed endotracheal intubation. After the third DC shock, we got the return of spontaneous circulation ROSC. The patient was transferred to the University hospital, were he was stabile, and PCI of the LAD was performed as per the standard protocol. Echocardiography performed in the CCU revealed hypokinesia of RV, with preserved systolic function. On hospital day 7 he had a full neurological recovery. He was conscious, oriented, with normal breathing, blood pressure 125/79 mmHg, sPO2 99, ECG: sinus rhythm, fr 87/min, without pathological signs. Echocardiography revealed the reduced systolic function of the left ventricle, with mitral regurgitation $\mathrm{MR}+2$.

Discussion: Out-of-hospital cardiac arrest (OHCA) is a major health problem in Europe and in the United States. The numbers of patients who have OHCA annually in these two parts of the world have traditionally been reported to be 275,000 and 420,000 respectively. The success of resuscitation depends on many factors: well-organized health care, organization of outpatient emergency services, but primarily when it comes to OHCA, education of the population on Basic life support, and early Cardiopulmonary resuscitation and use of AED (automated external defibrillator).
\end{abstract}

Keywords: Out-of hospital, cardiac arrest, Ventricular fibrillation, Cardiopulmonary resuscitation, ALS, BLS.

Original article, no submission or publication in advance or in parallel

* Corresponding author:

Kenan LJUHAR MD

$\triangle$ kenan.ljuhar@gmail.com

1 Emergency Medical Center of Canton Sarajevo, 71000 Sarajevo, BOSNIA and HERZEGOVINA

2 Emergency Clinic, University Clinical Centre of Kosovo, 10000 Pristina, Republic of KOSOVO

3 Department of General and Abdominal Surgery, University Medical Centre Maribor, Faculty of Medicine University of Maribor, 2000 Maribor, Republic of SLOVENIA

\section{Introduction}

Sudden OHCA (Out of hospital cardiac arrest) is the third leading cause of death in industrialized nations. In Europe, more than 350,000 patients are affected every year. And 100.000 could be saved if lay resuscitation - giving chestcompressions immediately and before the arrival of the emergency medical services (EMS) - was improved all over Europe.[1, 12]

In 2017 the American Heart Association (AHA) updated estimated the total annual burden of Out of Hospital Cardiac Arrest (OHCA) at 356,500. At least $23 \%$ of OHCA treated by Emergency Medical Service (EMS) have VF/VT as the initial rhythm.[2]

With more than $60 \%$ of cardiovascular deaths resulting from cardiac arrest, it remains the leading cause of death worldwide. [3] Cardiopulmonary resuscitation (CPR) can 
be defined as a set of measures and procedures performed in a patient who has experienced cardiac and/or respiratory arrest with the aim of restoring cardiac function and respiration. Successful resuscitation is considered to be the return of temporarily lost vital functions, primarily cardiac ROSC( return of spontaneous circulation).[4]

Heart rhythms associated with cardiac arrest are divided into two groups: shockable rhythms (ventricular fibrillation/pulseless ventricular tachycardia $(\mathrm{VF} / \mathrm{pVT})$ and non-shockable rhythms (asystole and pulseless electrical activity (PEA). The principal difference in the treatment of these two groups of arrhythmias is the need for attempted defibrillation in those patients with $\mathrm{VF} / \mathrm{pVT}$. Other interventions, including high-quality chest compressions with minimal interruptions, airway management, and ventilation, venous access, administration of adrenaline, and the identification and correction of reversible causes, are common to both groups. [5]

$\mathrm{VF}$ is the most commonly identified arrhythmia in cardiac arrest patients. The first monitored rhythm is ventricular fibrillation/pulseless ventricular tachycardia in approximately $20 \%$, both for in-hospital and out-of-hospital cardiac arrests.[6] The incidence of VF/pVT may be decreasing and can vary according to bystander CPR rates. Ventricular fibrillation/pulseless ventricular tachycardia will also occur at some stage during resuscitation in about $25 \%$ of cardiac arrests with an initial documented rhythm of asystole or PEA.[7] VF usually ends in death within minutes unless prompt corrective measures are instituted. $[1,2]$ Urgent medical treatment includes cardiopulmonary resuscitation and early defibrillation.

Protocols for cardiopulmonary resuscitation are the 2015 guidelines of the European Resuscitation Council (ERC) and the American Heart Association (AHA). Starting a survival chain is invaluable and increases the chances of successful resuscitation, with minimal deficits.

Ventricular fibrillation/ pulseless ventricular tachycardia as the initial rhythm has a better prognosis. Electrical defibrillation provides the single most important therapy for the treatment of these patients. [8]

\section{Material and Methods:}

For this case report are medical history from the resuscitation protocol of the Emergency Medical Center of Canton Sarajevo. The aim is to present a case of successful resuscitation in prehospital conditions, by the Emergency Medical Center of Canton Sarajevo, after ventricular fibrillation as initial rhythm.

Case report; Dispatch center of Emergency Medical Center of Canton Sarajevo received a call from a woman stating that her husband had fallen ill and that he had fainted. A team consisting of an emergency medicine doctor and two emergency medical technicians is sent from the central checkpoint. The team arrives at their home address in just under 5 minutes. There they find a 59-year-old man lying on the floor unconscious.

The patient is approached, and after checking the vital parameters, it is concluded that he is without consciousness, with no palpable carotid pulse, no spontaneous respirations, and wide non-reactive pupils. A postoperative scar is visible on the chest after heart surgery. Measures of Advanced life support, cardiopulmonary resuscitation, compression, and ventilation in a ratio of 30: 2 are approached, the airway is opened and the oropharyngeal tube is placed, and the patient is ventilated with a BMV with a maximum flow of $100 \% \mathrm{O} 2$ $15 \mathrm{~L} / \mathrm{min}$.

After placing ECG electrodes to the patient, the VF is read on the monitor as the initial rhythm (Figure 2), and immediately delivered DC Shock $200 \mathrm{~J}$ with pedals from the mobile Lifepack 15 defibrillator, and continued with CPR, with ventilation. An intravenous route is opened in a cubital fossa with an $18 \mathrm{G}$ diameter cannula, and a $500 \mathrm{ml} \mathrm{NaCl}$ $0.9 \%$ infusion solution is added. After 2 minutes of a 30 : 2 CPR cycle, the pulse is checked, also the rhythm on the monitor. The VF persists, and a second DC Shock 300J is delivered (Figure 3). Resuscitation measures are continued, Adrenaline $1 \mathrm{mg}$ is administered in a ratio of 1: 10000. The patient is intubated with tube number 7.5. After 2 minutes of $\mathrm{CPR}$, the pulse and rhythm are checked.

The monitor is still VF, and 3 DC Shock $360 \mathrm{~J}$ is

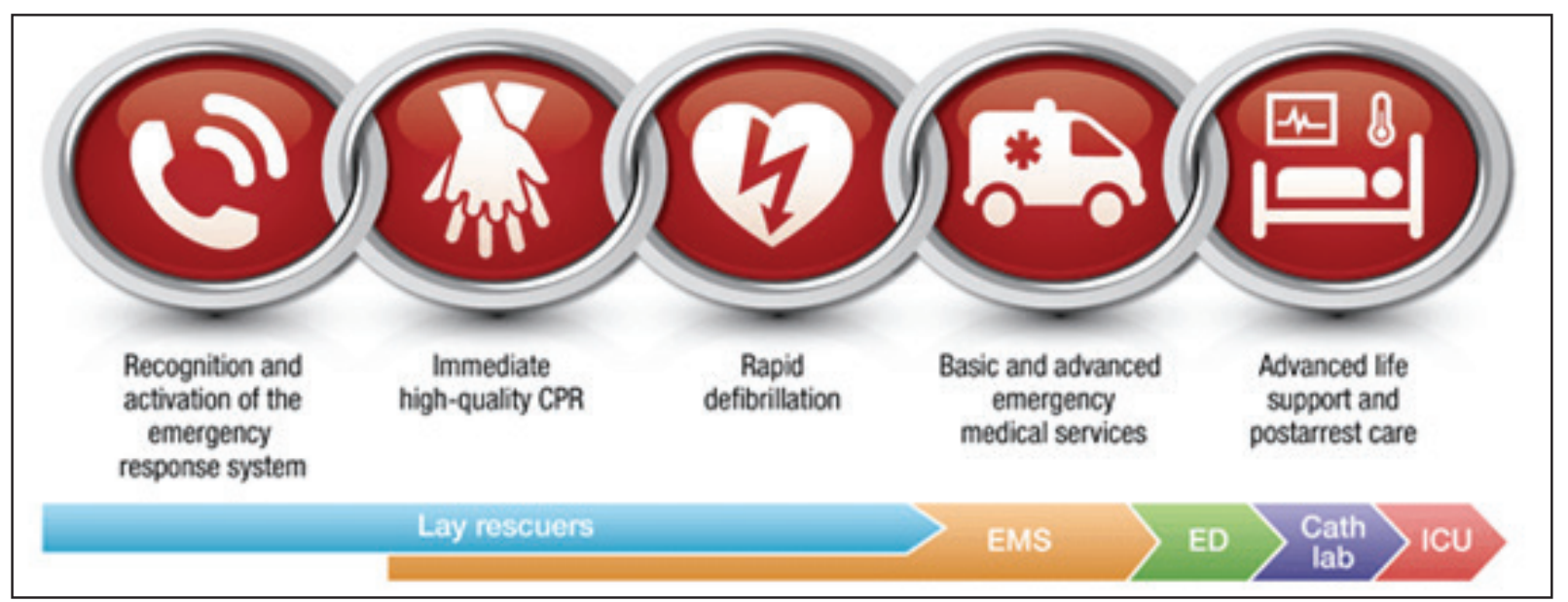

Figure 1: Chain of survival 


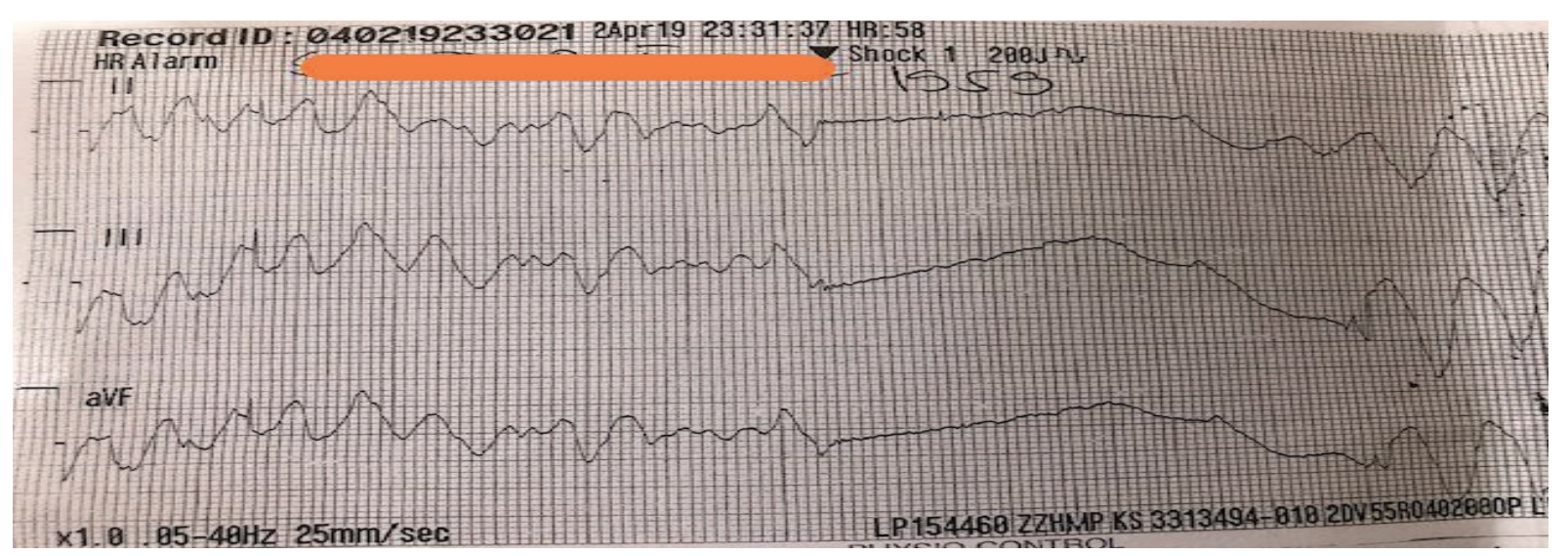

Figure 2: ECG -Initial rhythm VF,first DC Shock

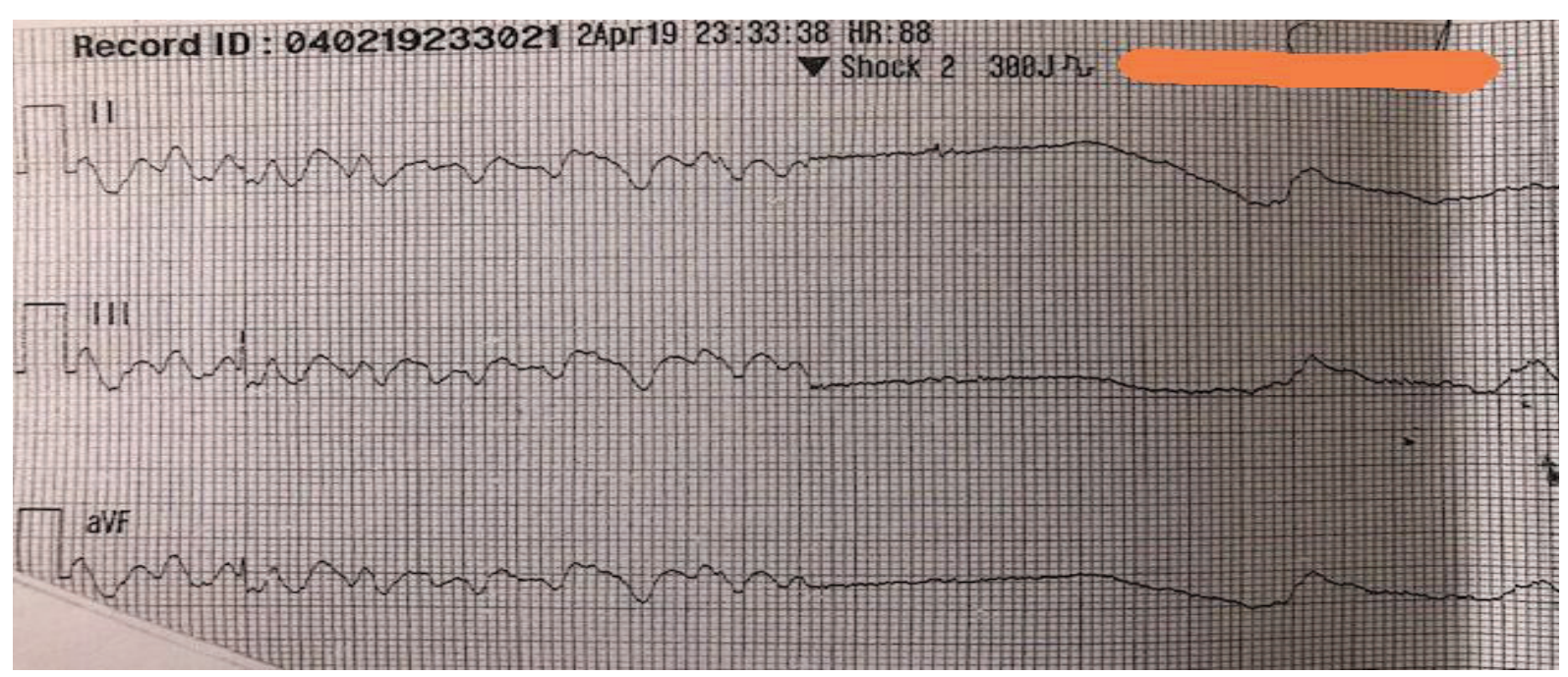

Figure 3: ECG after second DC Shock

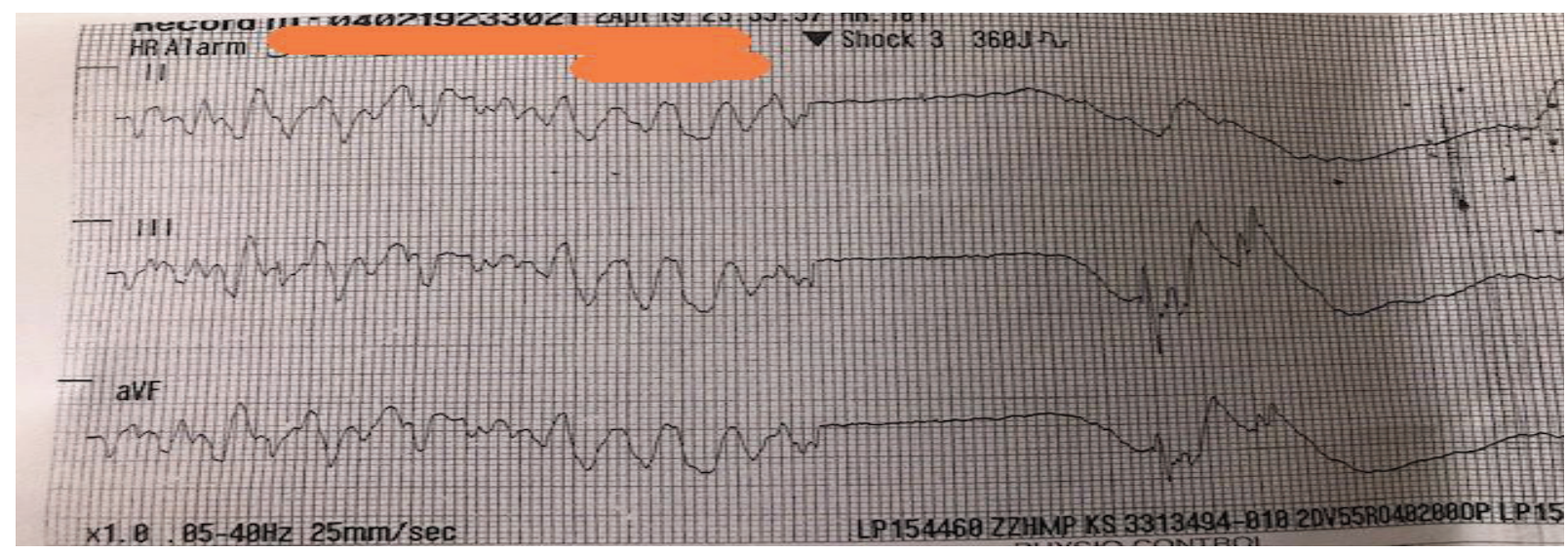

Figure 4:ECG after third DC Shock

delivered (Figure 4). A change occurs. A pulse is palpated over the a.Carotis, sinus rhythm fr $150 / \mathrm{min}$ on the monitor (Figure 5), peripheral pulse present, patients have about 8 spontaneous respirations. The patient is transferred with monitoring of vital parameters to the emergency aid circuit, where he connects to Medumat, an assisted breathing option. Objectively the patient remains unconscious, central and peripheral pulse present, blood pressure 110/70 $\mathrm{mmHg}$, saturation with sPO2 90, pupils react to light, about 10 respirations per minute, glucose $7.8 \mathrm{mmol} / \mathrm{L}$. On a 12-lead ECG sinus rhythm, fr 94 / min, with ST depression present in V2-V6 (Figure 6).

We learn from the family that he had heart surgery a month ago and that Bypass surgery was performed. Longtime cardiopathy. We transfer the patient to the University Clinical Center. The patient already starts 


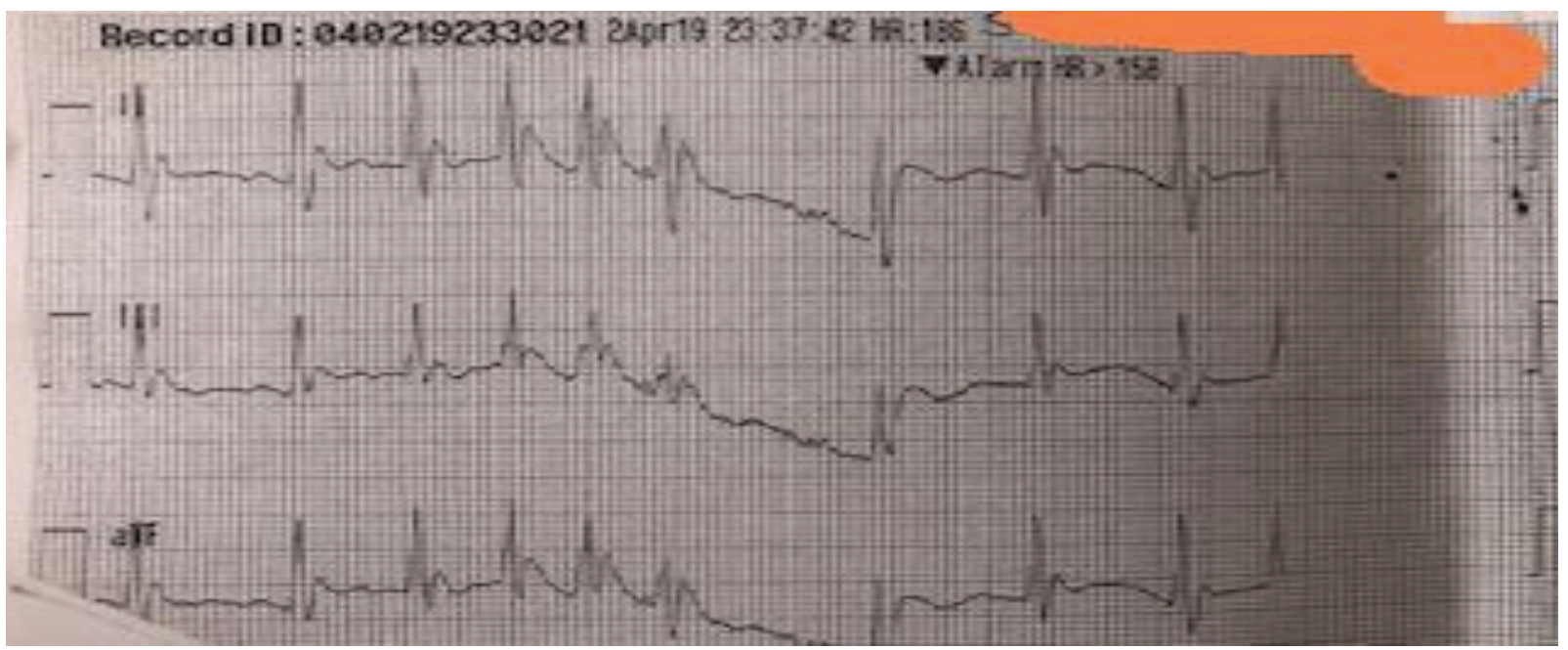

Figure 5:ECG after ROSC

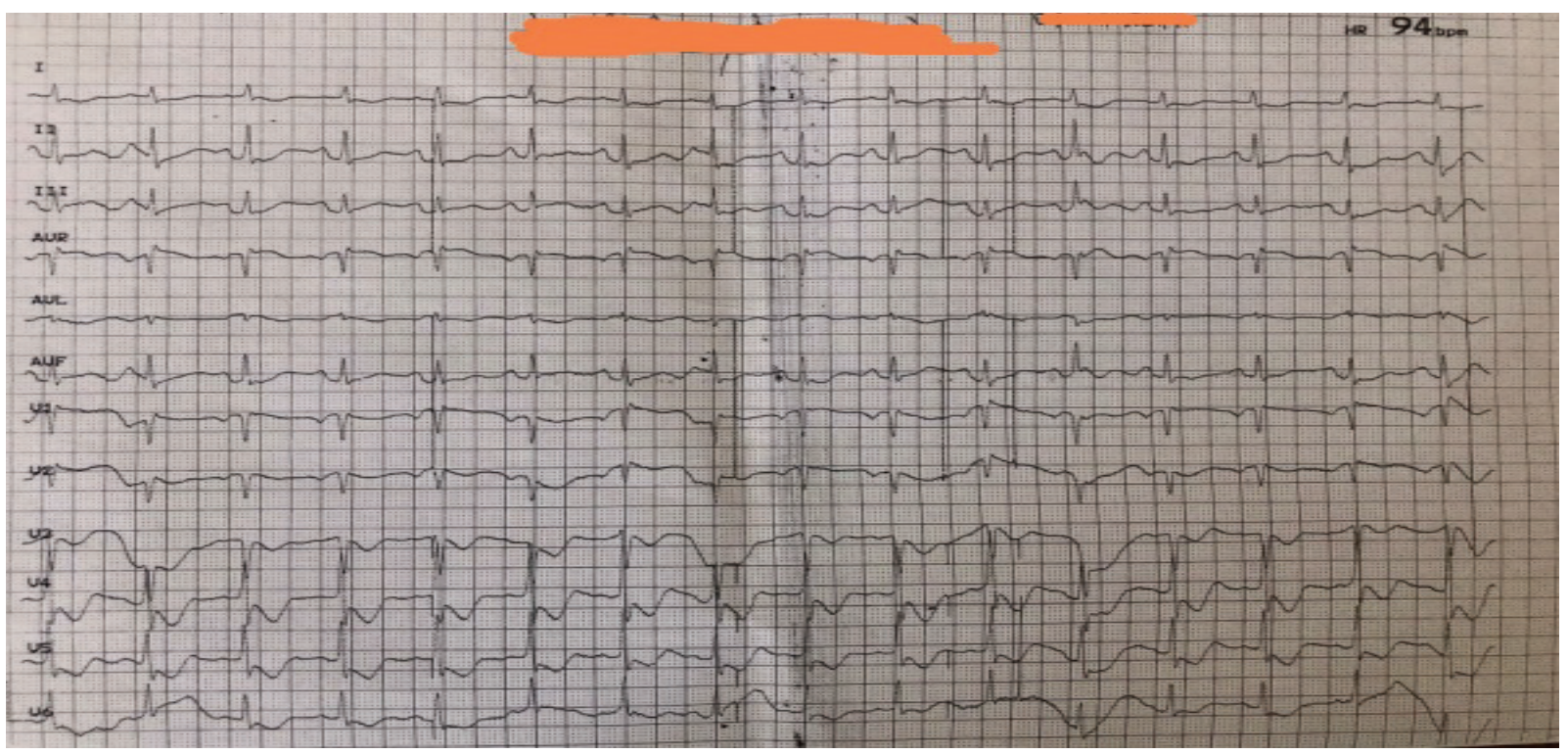

Figure 6: 12-lead ECG after ROSC

moaning, moves his arms, and tries to eject the endotracheal tube. The on-duty cardiologist and anesthesiologist place him in the cardiovascular care unit $\mathrm{CCU}$ and start preparations for the PCI procedure. PCI of the LAD was performed as per the standard protocol. Echocardiography performed in the CCU revealed hypokinesia of RV, with preserved systolic function. On hospital day 7 he had a full neurological recovery. He was conscious, oriented, with normal breathing, blood pressure 125/79mmHg, sPO2 99, ECG: sinus rhythm, fr $87 / \mathrm{min}$, without pathological signs. Echocardiography revealed the reduced systolic function of the left ventricle, with mitral regurgitation $\mathrm{MR}+2$.

\section{Discussion}

Out-of-hospital cardiac arrest (OHCA) is a major health problem in Europe and in the United States. The numbers of patients who have OHCA annually in these two parts of the world have traditionally been reported to be 275,000 and 420,000 respectively. 910 The success of resuscitation depends on many factors: well-organized health care, organization of outpatient emergency services, but primarily when it comes to OHCA, education of the population on Basic life support, and early Cardiopulmonary resuscitation and use of AED (automated external defibrillator). According to the American Heart Association's 2014 data, nearly 45 percent of out-of-hospital cardiac arrest victims survived when bystander CPR was administered. The majority of Out of Hospital Cardiac Arrests (OHCA) occurs in public settings (18.8 percent), mostly homes/residences $(69.5 \%)$ and nursing homes (11.7\%).11 Early CPR measures and early defibrillation are invaluable. To increase the number of effective cardiopulmonary resuscitation we need a CPR national and regional register, educated and wellequipped ambulance stationed AEDs. In some European countries, there are applications, with which we can see 
the network of AEDs in individual cities, where they are located. Educating the public about BLS (Basic life support) with the use of AEDs is crucial. The high success rate of resuscitation itself depends on the early CPR usually started by bystanders. Good organization of the emergency medical service, equipment, and education greatly contribute to a better outcome. The importance of the speed of arrival, which depends on the organization of the service itself, the dispatch center, and the deployment of teams in the city, is of great importance. Standardized equipment in all vehicles, and educated staff with the implementation of advanced life support measures.

\section{Conclusion}

Our case report showed successful resuscitation of OHCA in the patient's apartment. In this case, the ALS guidelines were applied. CPR was started immediately, after the detection of VF, defibrillation was delivered, and good ventilation and intubation of the patient ensured oxygenation. The speed of the team's arrival at the address, early CPR measures, early defibrillation, and airway management greatly contributed to the success of resuscitation. Transport to the University Hospital with monitoring of vital parameters and postresuscitation treatment enabled the patient to be discharged without any neurological sequelae in good psycho-physical conditions.

COI Statement: This paper has not been submitted in parallel. It has not been presented fully or partially at a meeting or podium or congress. It has not been published nor submitted for consideration beforehand.

This research received no specific grant from any funding agency in the public, commercial, or nonprofit sectors. There are no relevant or minor financial relationships from authors, their relatives or next of kin with external companies.

Disclosure: The authors declared no conflict of interest. No funding was received for this study.

\section{References:}

1. Nichol G., Thomas E., Callaway C.W., et al. Regional variation in out-of-hospital cardiac arrest incidence and outcome. JAMA. 2008;300:pp 1423-1431. [PMC free article] [PubMed] [Google Scholar]

2. Adabag AS, Luepker RV, Roger VL, Gersh BJ. Sudden cardiac death: epidemiology and risk factors. Nat Rev Cardiol. 2010 Apr;7(4): pp 216-25.[PMC free article] [PubMed]
3. Engdahl J, Holmberg M, Karlson BW, Luepker R, Herlitz J. The epidemiology of out-of-hospital 'sudden' cardiac arrest. Resuscitation. 2002;52:pp 235-245. [PubMed] [Google Scholar] [Ref list]

4. Nolan JP, Soar J, Zideman D, et al. 2010. European Council Guidelines for Resuscitation 2010. Section 1: Executive Summary. Resuscitation. 2010;81: pp 1219- 1276.

5. Jasmeet Soar, Jerry P. Nolanb, Bernd W. Böttiger, Gavin D. Perkins, Carsten Lott, Pierre Carli, Tommaso Pellis, Claudio Sandroni, Markus B. Skrifvars, Gary B. Smith, Kjetil Sunde, Charles D. Deakin, European Resuscitation Council Guidelines for Resuscitation 2015, Section 3. Adult advanced life support; Resuscitation 95 (2015), pp 100-147.

6. Steven M. Bradley, Erin E. Gabriel, Tom P. Aufderheide, Roxy Barnes, Jim Christenson, Daniel P. Davis, Ian G. Stiell, Graham Nichol, Survival Increases with CPR by Emergency Medical Services before defibrillation of out-ofhospital ventricular fibrillation or ventricular tachycardia: Observations from the Resuscitation Outcomes Consortium, Resuscitation, Volume 81, Issue 2,2010, pp 155-162.

7. Trond Nordseth, Theresa Mariero Olasveengen, Jan Terje Kvaløy, Lars Wik, Petter Andreas Steen, Eirik Skogvoll, Dynamic effects of adrenaline (epinephrine) in out-of-hospital cardiac arrest with initial pulseless electrical activity (PEA), Resuscitation, Volume 83, Issue 8,2012, pp 946-952.

8. Walter Kloeck, Richard O. Cummins, Douglas Chamberlain, Leo Bossaert, Victor Callanan, Pierre Carli, Jim Christenson, Brian Connolly, Joseph P. Ornato, Arthur Sanders, and Petter Steen, An Advisory Statement From the Advanced Life Support Working Group of the International Liaison Committee on Resuscitation, Vol 95, Issue 8, 1997;pp21832184.

9. Thomas D. Rea, Mickey S. Eisenberg, Greg Sinibaldi, Roger D. White; Incidence of EMS-treated out-of-hospital cardiac arrest in the United States; Resuscitation; Volume 63, Issue1,2004,pp17-24.

10. Christie Atwood, Mickey S. Eisenberg, Johan Herlitz, Thomas D. Rea; Incidence of EMS-treated out-of-hospital cardiac arrest in Europe; Resuscitation; Volume 67, Issue 1;2005; pp 75-80.

11. Mozaffarian D, Benjamin EJ, Go AS, et al. on behalf of the American Heart Association Statistics Committee and Stroke Statistics Subcommittee. Heart disease and stroke statistics - 2015 update: a report from the American Heart Association. Circulation. 2015;131(4):e29-e322. [PubMed] [Google Scholar]

12. https://www.erc.edu/projects/eureka-one 